\title{
Diagnosing fracture-related infections: can we optimize our nuclear imaging techniques?
}

\author{
Andor W. J. M. Glaudemans ${ }^{1}$ (D) Paul Bosch ${ }^{2} \cdot$ Riemer H. J. A. Slart $^{1,3} \cdot$ Frank F. A. IJpma $^{2} \cdot$ Geertje A. M. Govaert $^{4}$
}

Received: 13 May 2019 / Accepted: 27 May 2019 / Published online: 12 June 2019

(C) Springer-Verlag GmbH Germany, part of Springer Nature 2019

"It is difficult to treat a disease that has not been properly diagnosed"

Fracture-related infection (FRI) is one of the most serious problems in orthopaedic care. The incidence of FRI is estimated to be $3 \%$, but may be as high as $45 \%$, depending on comorbidities (diabetes, vascular disease, smoking) and the extent of the injury (presence of severe soft tissue injuries). FRI has a huge impact on the patient's daily life, since this condition often requires a lengthy treatment regime including multiple reoperations and prolonged antibiotic treatment, and could even require limb amputation.

It is challenging to diagnose FRI, because its clinical manifestations are quite variable. In some cases the clinical scenario is clear and FRI can be diagnosed by physical examination only, e.g. in the case of wound dehiscence with an exposed osteosynthesis implant. It is also possible that the classic symptoms of infection, such as redness, swelling and/or pain, are absent. For the physician, it is often a challenge to discriminate bacterial infections from the inflammatory responses to traumatic soft tissue damage, (delayed) fracture

This article is part of the Topical Collection on Orthopaedics

Andor W. J. M. Glaudemans

a.w.j.m.glaudemans@umcg.nl

1 Medical Imaging Center, Department of Nuclear Medicine and Molecular Imaging, University of Groningen, University Medical Center Groningen, Hanzeplein 1, 9700

RB Groningen, The Netherlands

2 Department of Trauma Surgery, University of Groningen, University Medical Center Groningen, Groningen, The Netherlands

3 TechMed Centre, Department of Biomedical Photonic Imaging, University of Twente, Enschede, The Netherlands

4 Department of Trauma Surgery, Utrecht University, University Medical Center Utrecht, Utrecht, The Netherlands healing, previous surgery and posttraumatic arthrosis. In these circumstances, different imaging techniques may contribute to the diagnostic process. In general, three clinical issues may require medical imaging and should be addressed in the diagnostic workup of a suspected fracture-related infection:

1. To evaluate fracture consolidation and stability of any implants

2. To detect and locate a potential infection

3. To image the extent of the infection and specify anatomic characteristics for surgical planning

There are several types of imaging modalitiesy that may be preferred and the choice often depends on local expertise, costs and availability. The most commonly used techniques (singly or in combination) are plain radiography, computed tomography (CT), magnetic resonance imaging (MRI), three-phase bone scintigraphy (TPBS), white blood cell (WBC) or antigranulocyte antibody scintigraphy with single photon emission computed tomography (SPECT/CT) and ${ }^{18}$ F-fluorodeoxyglucose positron emission tomography combined with low-dose or contrast-enhanced CT (FDG PET/CT) [1-3]. It is acknowledged in the literature that the optimal diagnostic strategy for FRI remains unclear [3-5]. A survey on preferred diagnostic imaging strategies conducted amongst 346 medical specialists (trauma surgeons, orthopaedic surgeons, nuclear medicine physicians and radiologists found a significant difference among the specialists and poor availability of local protocols to diagnose and treat FRI [5].

Until recently, the available recommendations for the diagnostic workup of FRI were based on case reports, retrospective series, expert opinion and local consensus meetings [6-8]. It is also important to state that the diagnostic principles that apply to a prosthetic joint infection (PJI) cannot be extrapolated one-on-one to FRI. Fracture fixation behaves differently from an arthroplasty (e.g. in terms of stability), and increased bone metabolism occurs during fracture healing, which may influence image quality and mimic the appearance of infection on imaging. 
Recently, a consensus document from four European societies, including the EANM, the European Society of Radiology (ESR), the European Bone and Joint Infection Society (EBJIS) and the European Society of Clinical Microbiology and Infectious Diseases (ESCMID), was published. It provides levels of evidence for each diagnostic modality and a commonly agreed diagnostic flow chart [3]. However, the available evidence was considered to be limited, and large multicentre prospective studies comparing the different imaging modalities are needed.

Another problem when interpreting early literature is the heterogeneity among included patient groups. Until recently, many different definitions of infection following surgical fracture care were used. Terms such as osteitis, osteomyelitis, posttraumatic osteomyelitis, implant-related infection, deep or superficial surgical site infection and many more were applied without a uniform agreement on an actual definition of this complication [9]. Over the past few years there has been a growing awareness of the need for a widely supported consensus on the definition of infection after fracture care similar to that already established for PJI [10]. This will allow physicians to compare the results of future research and the subsequent development of international protocols and guidelines. Last year, a consensus definition was published by a group of experts representing the Association for the Study of Internal Fixation (Arbeitsgemeinschaft für Osteosynthesefragen, AO), the EBJIS, and representatives from prominent orthopaedic trauma hospitals and academic centres [11]. According to this definition, the term "fracture-related infection (FRI)" is considered to encompass the complete spectrum of infections (e.g. acute and chronic, superficial and deep, with and without bone involvement, with and without implants in situ) following surgical fixation of closed or open fractures. Two levels of certainty were defined around the diagnostic features of FRI. Criteria could be classified as confirmatory (infection definitely present) or suggestive (infection possibly present). Radiological and/or nuclear imaging signs are considered one of the suggestive criteria in the diagnosis of FRI [11].

Focusing on the nuclear imaging modalities, TPBS plays a limited role and can only be used as a first screening method to exclude the presence of FRI in patients with a low probability of infection [3]. WBC scintigraphy and FDG PET provide higher accuracy for diagnosing FRI. Recently, two studies in a large homogeneous patient group with suspected FRI have been published [12, 13]. In the first study [12], WBC scintigraphy was performed (using the correct dual time point imaging with decay correction, and the correct interpretation criteria according to EANM protocols $[14,15])$ in 192 consecutive patients. The reference standard was based on the outcome of microbiology in patients undergoing surgery or on clinical follow-up of at least 6 months. WBC scintigraphy was found to have a sensitivity of $79 \%$, a specificity of $97 \%$, a positive predictive value of $91 \%$, a negative predictive value of $93 \%$ and a diagnostic accuracy of $92 \%$ for detecting FRI in the peripheral skeleton. Three other important findings were mentioned: (1) the time between surgery and WBC scintigraphy did not influence its diagnostic accuracy, (2) the concomitant use of antibiotics and/or NSAIDs did not influence its diagnostic accuracy, and (3) the majority of patients with a false-negative scan had a low-grade infected nonunion [14].

In the second retrospective cohort study, 156 FDG PET/CT scans were performed in 135 patients with suspected FRI [13]. Besides the visual analysis (uptake location, uptake pattern, and grade of uptake), semiquantitative evaluation was also performed by measuring standardized uptake values (SUVs) on EARL reconstruction scans. The reference standard was the microbiology results from at least two per-operative deep tissue cultures or the presence/absence of clinical confirmatory signs of FRI (conforming to the previously mentioned FRI consensus definition) during a follow-up of at least 6 months. Visual assessment of the scans showed a sensitivity of $89 \%$, a specificity of $80 \%$, a positive predictive value of $74 \%$, a negative predictive value of $91 \%$ and a diagnostic accuracy of $83 \%$. SUVs on their own showed a lower diagnostic performance, but combining them with visual assessment yielded a somewhat higher area under the curve than visual assessment alone. Another important finding was that a time between surgery and the FDG PET/CT scan of less than 1 month was associated with a significant increase in false-positive scan results [15].

Although the diagnostic accuracy of WBC scintigraphy has been found to be slightly higher, FDG PET/CT is also a good diagnostic tool in patients with suspected FRI, because of its wider availability and more convenience for the patient. Furthermore, the newer hybrid camera systems have a better spatial resolution and their CT component is less likely to be affected by metal artefacts, which may lead to further improvement in the diagnostic accuracy of FDG PET/CT in patients with suspected FRI.

In the cited studies, the patient populations studied showed good homogeneity, but still all patients with suspected FRI were clustered. In the study by Sollini et al. in this issue, the diagnostic performance of FDG PET/CT was assessed in a specific group of 47 patients with nonunion [16]. In the treatment of nonunion, it is essential to determine if an underlying infectious process is the reason of the occurrence of impaired bone healing. The authors used strict criteria for the visual analysis of both the FDG PET scan and the CT scan. The three FDG PET criteria for positivity were: (1) asymmetrical FDG uptake in the nonunion region compared to the contralateral area, (2) distinguished areas of focal FDG uptake involving bone fragments of the nonunion, and (3) increased diffuse uptake along the bone-metallic device or bone-bone or bone-graft surfaces. FDG PET/CT was considered positive when at least one PET criterion and one $\mathrm{CT}$ criterion were present. In total, 22 patients had a noninfected nonunion, 
whereas 25 had positive intraoperative microbiological results. Infection was correctly detected on visual analysis of FDG PET/CT scans in 23 patients, whereas two patients showed no increased FDG uptake and the scans were considered false-negative (diagnostic accuracy $81 \%$ ). These results are consistent with those of the previous study (diagnostic accuracy $83 \%$ ) by Lemans et al. [13]. The authors concluded that FDG PET/CT is a promising tool for the specific evaluation of infected nonunion [16].

Our understanding of the value of nuclear imaging techniques, including hybrid techniques, in patients with FRI has significantly increased over the last years, and they often provide information essential to the decision-making process of the (orthopaedic) trauma surgeon. Figure 1 shows different imaging features of FRI that may affect the treatment strategy. The presence of fistula, sinus, wound breakdown and pus are considered confirmatory signs of FRI [11], which need surgical treatment (Fig. 1a, b). In other cases, the presence of FRI could be less obvious (suggestive criteria), for example pain, swelling, impaired bone healing, implant failure, slightly elevated serum inflammatory markers [17], or persistent wound drainage [11]. First, a CT scan is usually performed (if possible as a hybrid technique with other imaging modalities) to assess whether the fracture has healed (Fig. 1c-e) and the potentially infected implant (e.g. plate, screws and/or nail) can be safely removed. Second, additional imaging techniques have the ability to detect or rule out FRI and subsequently determine the extent of the infection (Fig. 1f-j). Establishing or ruling out FRI by WBC scintigraphy with SPECT/CT, FDG $\mathrm{PET} / \mathrm{CT}$ or MRI could avoid unnecessary reoperations. A positive scan result will lead the surgeon to alter the treatment strategy, e.g. to a two-stage instead of one-stage revision surgery for an infected nonunion. The first stage, known as Masquelet technique, consists of removal of infected tissue, if indicated placement of a temporary spacer followed by antibiotic treatment for several weeks. The second stage consists of the final reconstruction and fracture fixation. Finally, visualization of the extent of an FRI will help the surgeon preoperatively plan the surgical approach and extent of debridement.

Although the first steps have been taken, more work needs to be done on optimizing imaging modalities. Recently, a prospective diagnostic imaging trial in patients with FRI has started in which the most common types of advanced medical imaging (MRI, FDG PET and WBC scintigraphy) will be

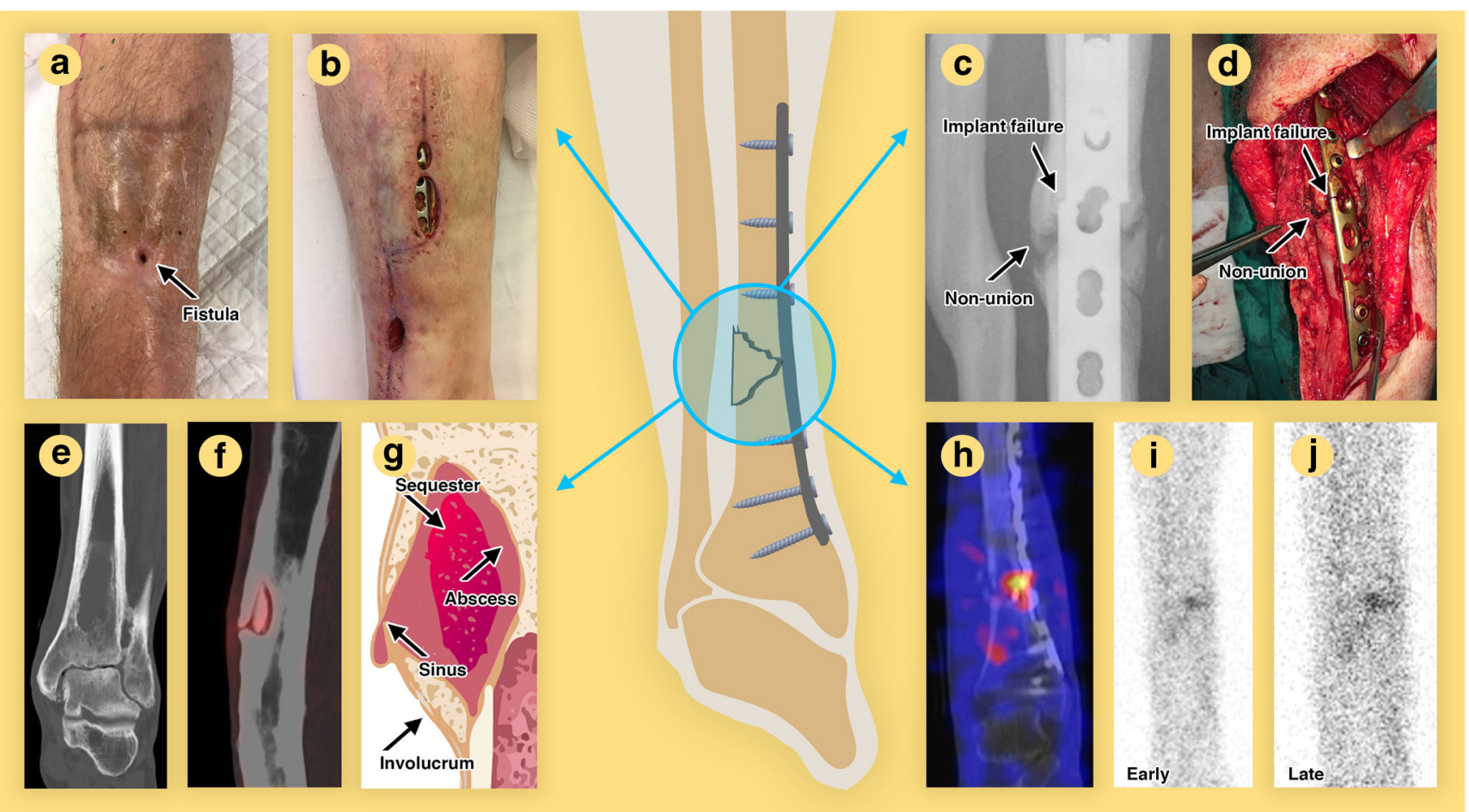

Fig. 1 Clinical, radiological, surgical and nuclear imaging features of fracture-related infections. a Fistula which is a communication with the bone or implant. b Wound breakdown with exposed implant. c Plain radiograph showing a nonunion with implant failure, which is considered an FRI. d Intraoperative view of an infected nonunion with plate breakage. e CT image of a subacute intraosseous FRI after fracture treatment, known as Brodie's abscess. f FDG PET/CT image of an encapsulated sequestrum (piece of dead bone which often maintains an FRI). $\mathbf{g}$
Schematic representation of the sequestrum surrounded by an abscess together with a sinus tract to the soft tissues and involucrum (periosteal bone formation caused by pus accumulation below the periosteum) of the bone. $\mathbf{h ~ W B C ~ S P E C T / C T ~ i m a g e ~ s h o w s ~ a ~ h o t s p o t ~ a t ~ t h e ~ f r a c t u r e ~ s i t e ~ a f t e r ~}$ plate osteosynthesis for the distal tibial fracture. I, $\mathbf{j}$ Dual time point WBC scintigraphy with ${ }^{99 \mathrm{~m}} \mathrm{Tc}-\mathrm{HMPAO}$-labelled autologous leucocytes showing (I) uptake in the early phase and (j) an increase in intensity in the late phase, which is a clear sign of infection 
directly compared in terms of their diagnostic accuracy and ability to display surgically relevant details [18]. This should result in a standardized approach in determining the best diagnostic imaging strategy in patients with suspected FRI, and the implementation of this knowledge in evidence-based national and international guidelines.

However, there are still some issues in patients with FRI that should be further investigated. Infected implants are covered by a biofilm, defined as a microbial community composed of cells producing an extracellular polymer matrix that protects them from the immune system. Will we be able to detect and visualize the maturation of these biofilms in the future? Until now we have not been able to visualize the biofilm properly due to: (1) changes in properties (e.g. phenotype, rate of growth and gene transcription) of the bacteria that stick to the surface of biomaterials, implants and devices, (2) poor penetration of tracers into the biofilm, (3) the often low amount of bacteria involved in biofilms, and (4) the limited spatial resolution of the imaging devices.

Future research into nuclear medicine imaging techniques should also focus on the possibility of detecting the particular microorganism that has caused the infection. In the past, some attempts were made with radiolabelled antibiotics, such as ciprofloxacin, but without satisfactory results [19]. In our opinion, following recent developments in available camera systems, now might be the right time to focus on the early detection of the involved bacteria to allow early adjustment of antibiotics to improve overall outcomes. Not only is this early identification important, but detection of specific resistance mechanisms (such as the thickness and maturation of the biofilm) might also be a clear goal, since the incidence of antibiotic resistance is increasing, and infection prevention is an ultimate goal for the community. Furthermore, FRI imaging is not limited to standard imaging devices. Optical imaging and optoacoustic imaging with specific fluorescent tracers could also be useful, although these techniques are still limited by their maximum penetration depth. Perhaps dual probes both for optical and for PET imaging - could be the solution.

In summary, major steps have been made in recent years by establishing a consensus definition of FRI, and also in gathering evidence regarding the application of nuclear imaging techniques in this difficult-to-image patient population. However, we still need to optimize our existing diagnostic procedures, we need strict criteria to call a scan positive for FRI, and we need large prospective trials in which the available techniques will be compared directly with each other. We also have to develop innovative techniques such as specific bacterial tracers, including imaging techniques that are able to detect biofilm specifics, bone viability, and antimicrobial resistance against antibiotics. By implementing all these new tools in the near future, we might be able to provide exact information on the presence, location and extent of a fracture-related infection to the trauma and orthopaedic surgeons.

\section{Compliance with ethical standards}

Ethical approval All procedures performed in studies involving human participants were in accordance with the ethical standards of the institutional committee (University Medical Center Groningen) and with the principles of the 1964 Declaration of Helsinki and its later amendments or comparable ethical standards.

Informed consent Informed consent was obtained from the individual included in Fig. 1 of this paper.

Conflicts of interest None.

\section{References}

1. Govaert GA, Glaudemans AW. Nuclear medicine imaging of posttraumatic osteomyelitis. Eur J Trauma Emerg Surg. 2016;42(4): 397-410.

2. Govaert GA, IJpma FFA, McNally M, McNally E, Reininga IH, Glaudemans AW. Accuracy of diagnostic imaging modalities for peripheral post-traumatic osteomyelitis - a systematic review of the recent literature. Eur J Nucl Med Mol Imaging. 2017;44(8):1393407.

3. Glaudemans AW, Jutte PC, Cataldo MA, Cassar-Pullicino V, Gheysens O, Borens O, et al. Consensus document for the diagnosis of peripheral bone infection in adults: a joint paper by the EANM, EBJIS, and ESR (with ESCMID endorsement). Eur J Nucl Med Mol Imaging. 2019;46(4):957-70.

4. Morgenstern M, Kuhl R, Eckardt H, Acklin Y, Stanic B, Garcia M, et al. Diagnostic challenges and future perspectives in fracturerelated infection. Injury. 2018;49(Suppl 1):S83-90.

5. Govaert GA, Glaudemans AW, Ploegmakers JJ, Viddeleer AR, Wendt KW, Reininga IHF. Diagnostic strategies for posttraumatic osteomyelitis: a survey amongst Dutch medical specialists demonstrates the need for a consensus protocol. Eur J Trauma Emerg Surg. 2018;44(3):417-26.

6. Zimmerli W. Clinical presentation and treatment of orthopaedic implant-associated infection. J Intern Med. 2014;276(2):111-9.

7. Hake ME, Oh JK, Kim JW, Ziran B, Smith W, Hak D, et al. Difficulties and challenges to diagnose and treat post-traumatic long bone osteomyelitis. Eur J Orthopaed Surg Traumatol. 2015;25(1):1-3.

8. Jutte P, Lazzeri E, Sconfienza LM, Cassar-Pullicino V, Trampuz A, Petrosillo N, et al. Diagnostic flowcharts in osteomyelitis, spondylodiscitis and prosthetic joint infection. Q J Nucl Med Mol Imaging. 2014;58(1):2-19.

9. Morgenstern M, Moriarty TF, Kuehl R, Richards RG, McNally MA, Verhofstad MHJ, et al. International survey among orthopaedic trauma surgeons: lack of a definition of fracture-related infection. Injury. 2018;49(3):491-6.

10. Parvizi J, Zmistowski B, Berbari EF, Bauer TW, Springer BD, Della Valle CJ, et al. New definition for periprosthetic joint infection: from the Workgroup of the Musculoskeletal Infection Society. Clin Orthop Relat Res. 2011;469(11):2992-4.

11. Metsemakers WJ, Morgenstern M, McNally MA, Moriarty TF, McFadyen I, Scarborough M, et al. Fracture-related infection: a consensus on definition from an international expert group. Injury. 2018;49(3):505-10.

12. Govaert GA, Bosch P, IJpma FF, Glauche J, Jutte PC, Lemans JV, et al. High diagnostic accuracy of white blood cell scintigraphy for fracture related infections: results of a large retrospective singlecenter study. Injury. 2018;49(6):1085-90. 
13. Lemans JV, Hobbelink MG, IJpma FF, Plate JDJ, van den Kieboom $\mathrm{J}$, Bosch P, et al. The diagnostic accuracy of 18F-FDG PET/CT in diagnosing fracture-related infections. Eur J Nucl Med Mol Imaging. 2019;46(4):999-1008.

14. Glaudemans AW, de Vries EF, Vermeulen LE, Slart RH, Dierckx RA, Signore A. A large retrospective single-centre study to define the best image acquisition protocols and interpretation criteria for white blood cell scintigraphy with 99mTc-HMPAO-labelled leukocytes in musculoskeletal infections. Eur J Nucl Med Mol Imaging. 2013;40(11):1760-9.

15. Signore A, Jamar F, Israel O, Buscombe J, Martin-Comin J, Lazzeri E. Clinical indications, image acquisition and data interpretation for white blood cells and anti-granulocyte monoclonal antibody scintigraphy: an EANM procedural guideline. Eur J Nucl Med Mol Imaging. 2018;45(10):1816-31.

16. Sollini M, Trenti N, Malagoli E, Catalano M, Di Mento L, Kirienko $\mathrm{A}$, et al. [18F]FDG PET/CT in non-union: improving the diagnostic performances by using both PET and CT criteria. Eur J Nucl Med Mol Imaging. 2019. https://doi.org/10.1007/s00259-019-04336-1.

17. Bosch P, van den Kieboom J, Plate JDJ, IJpma FF, Houwert RM, Huisman A, et al. Limited predictive value of serum inflammatory markers for diagnosing fracture-related infections: results of a large retrospective multicenter cohort study. J Bone Jt Infect. 2018;6(3): $130-7$.

18. Govaert GAM, Hobbelink MGG, Reininga IHF, Bosch P, Kwee $\mathrm{TC}$, de Jong PA, et al. The accuracy of diagnostic imaging techniques in patients with a suspected fracture-related infection (IFI) trial: study protocol for a prospective multicenter cohort study. Submitted.

19. Glaudemans AW, Slart RH, van Dijl JM, van Oosten M, van Dam GM. Molecular imaging of infectious and inflammatory disease: a terra incognita. J Nucl Med. 2016;56(5):659-61.

Publisher's note Springer Nature remains neutral with regard to jurisdictional claims in published maps and institutional affiliations. 